Journal of Engineering and Applied Sciences 14 (17): 6282-6290, 2019

ISSN: 1816-949X

(C) Medwell Journals, 2019

\title{
Color Identification in Aminobenzenes with Voltage Values Obtained from Transmittance and Absorbance
}

\author{
${ }^{1}$ L. Ivan C. Bravo, ${ }^{1}$ Fredy E. Hoyos and ${ }^{2}$ John E. Candelo-Becerra \\ ${ }^{1}$ Facultad de Ciencias, Escuela de Física, Universidad Nacional de Colombia, Sede Medellín \\ Calle 59 A No. 63-20, Medellín 050034, Antioquia, Colombia \\ ${ }^{2}$ Departamento de Energía Eléctrica y Automática, Facultad de Minas, \\ Universidad Nacional de Colombia, Sede Medellín, Carrera 80 No. 65-223, \\ 050041 Medellín, Colombia, fehoyosve@unal.edu.co,+57-4-4309000
}

\begin{abstract}
This study presents electronic spectrophotometry to measure the transmittance and absorbance of aminobenzene that allows identifying the color of the substance. Voltage signals are measured from the amount of light that passes each quartz sample and the values are compared with the wavelengths of the visible spectrum impact. The results show that each substance produces a specific voltage signal in a color range similar to that of the sample and a particular waveform. Both the transmittance and absorbance are identified with the spectrophotometer once the voltage ratio that produces each of the substances in the sample is identified. All the voltages and wavelength generated with each quartz sample testing each color is included in the study.
\end{abstract}

$\underline{\text { Key words: Color identification, aminobenzenes, transmittance, absorbance, spectrophotometer, voltage signals }}$

\section{INTRODUCTION}

The colors of a solution sample can be identified by using the concept of transmittance and absorbance. Transmittance can be defined as the amount of light that crosses a substance or a body in a quantity of time and the absorbance as the amount of light that absorbs that body. Transmittance or the absorbance can be measured by spectrophotometry which is one of the primary scientific methods used to identify substances. Several researches have been presented using spectrophotometers for the identification of substances in scientific, medical and industrial fields. By Takamoto et al. (2013) oral mucosal diseases are identified by a portable spectrophotometer, comparing the different colors and epithelial thickness as an essential finding the spectrophotometer could measure a slight color change of a white lesion unrecognizable to the human eyes. From spectrophotometry, other more complex methods for the analysis of substances and compounds are derived such as the flux spectrophotometry which analyzes substances that are in continuous movements and High-Performance Liquid Chromatography (HPLC). That separates compounds from mixtures for further analysis. By Petruci et al. (2017), a flow-through optical absorption detector for HPLC using a deep-UV light-emitting diode with an emission band at $235 \mathrm{~nm}$ is presented which is coupled to a system HPLC and the determination of the antidiabetic drugs pioglitazone was successfully.

Spectrophotometry is also widely used in biochemistry studies. By Rojas et al. (2013), the determination of species and the study of biochemical processes using UV spectrophotometry-visible is presented. The micromolar concentrations of substances can be determined using this technique. This technique often presents interferences in the measurements of the compounds of interest but they are minimized by means of differential or derivative spectrophotometry.

Another important topic is the mass spectrophotometry which consists of the molecular particle separation by their different mass. By Burnum-Johnson et al. (2017), the researchers explain the main factors of a successful pregnancy and the necessity of a better comprehension of the molecular changes associated to those for the correct treatment, if a problem in this factor is presented. The needs are due to the limitation of the conventional techniques as the magnetic resonance. The mass spectrophotometry has been used to identify metabolic and lipids associated with

Corresponding Author: Fredy E. Hoyos, Facultad de Ciencias, Escuela de Física, Sede Medellín, Universidad Nacional de Colombia, Calle 59 A No. 63-20, 050034 Medellín, Colombia fehoyosve@unal.edu.co,+57-4-4309000 
complications related to pregnancy. As mentioned above, mass spectrophotometry is of great importance in many fields, among this medicine, the food industry, metals, perfumes, environmental studies, chemical and biochemical studies. According to Gloess et al. (2018), the researchers present an online analysis of coffee roasting using Ion Mobility Spectrometry-Mass Spectrometry (IMS-MS) with corona discharge ionization. This is the first time that the formation of Volatile Organic Compounds (VOCs) during coffee roasting it is monitored in positive and negative ion mode and not only with mass spectrometry but also with ion mobility spectrometry, the latter allowed the separation of isobaric and isomeric compounds. In positive ion mode, the isomers of the alkylpyrazines exhibited different intensity profiles during the roasting process. In negative ion mode, it allowed access to species hardly detectable by other online methods such as acids.

Mass spectrometry also plays an essential role in the field of biology and medicine. By Garrett (2017), the researcher talk about how by using mass spectrometry the lipids are analyzed, especially those isolated from bacteria. Through these studies it has been possible to identify new lipid structures, several new lipids of Gram-positive and Gram-negative bacteria are presented. Another process similar to the HPLC by Petruci et al. (2017), the LC-MS (Liquid Chromatography and Mass Spectrometry) which separates mixtures into its components and by means of spectrometry to analyze these components separately. By Ketha et al. (2017) explain how this process has become a powerful tool in the field of endocrinology.

During the last 30 years, clinical LC-MS applications led to several clinical advances: diagnostic sensitivity of newborn screening for Congenital Adrenal Hyperplasia $(\mathrm{CAH})$, inaccuracy of sex steroid measurements especially at low concentrations, in pheochromocytoma diagnosis and in vitamin $\mathrm{D}$ metabolite testing. Essays for protein biomarkers including Insulin-like Growth Factor 1 (IGF-1) and Angiotensin I (AngI) for Plasma Renin Activity (PRA) measurement have been introduced for clinical use.

This technique is very efficient to measure the absorbance and transmittance. However, these instruments have high prices on the market. Most of the experiments seek to measure absorbance in different concentrations of substances but no comparisons of various pigments and the voltages generated by a sensor are performed considering the same concentration.

Therefore, it is important to find a direct relationship between light intensity and the voltage measured with the light sensor, to obtain a measure of absorbance more simply compared with the high-cost commercial spectrophotometers. This research study is based on the comparison of the absorbance and transmittance of water with various aminobenzene using a voltage signal of low-cost spectrophotometry. The main characteristics of the method are the construction of lowcost spectrophotometry that measures homogenous samples, the comparison of the luminous intensity that impacts a light sensor and the voltage measured with the spectrophotometer, comparison between absorbances of aminobenzene of different colors using the same concentration in the solution.

\section{MATERIALS AND METHODS}

In this study, first the mathematics of the lenses, diffraction, transmittance and transmittance are presented. Besides, a description of the sections and the material required to construct the spectrophotometers are presented.

Figure 1 shows the complete system used for the spectrophotometry tests. This system has an electronic circuit designed to move an LED through a servomotor as shown in the upper left of Fig. 1. Thus, a potentiometer is used to supply an LM 555 with a variable voltage signal which allows generating of the PWM signal that moves the servomotor and changes the position of the light that the LED emits. Then, the light is concentrated by a diffraction grid that impacts the samples of different colors. Furthermore, another electronic circuit was designed as shown to the right of Fig. 1, to measure the light intensity with a photodiode which converts the light to voltage signals that are finally amplified to identify the response of the different colors of the samples.

Lens: Figure 2 shows the lens and the distance required for the spectrophotometer. In Fig. 2a, the term $O$ represents the object, $I$ is the virtual image of the object generated by the lens, $\mathrm{p}$ is the distance from the lens to the object, $q$ is the distance from the lens to the image and $R_{1}$ and $R_{2}$ are the radios of the lens with $\left(R_{1}=R_{2}\right)$. Besides, $C_{1}$ is the distance from the lens to the image and $C_{2}$ is the distance from the lens to the object. Once, the light strikes on one side of the lens, beams concentrate in one point, depending on the distance $\mathrm{C}_{2}$ that the object is located. For the case presented in this study, the light is produced in a power LED and pointed it out directly to the lens as a flat waveform front coming from the infinity, so, the lens has the effects shown in Fig. $2 \mathrm{~b}$. 


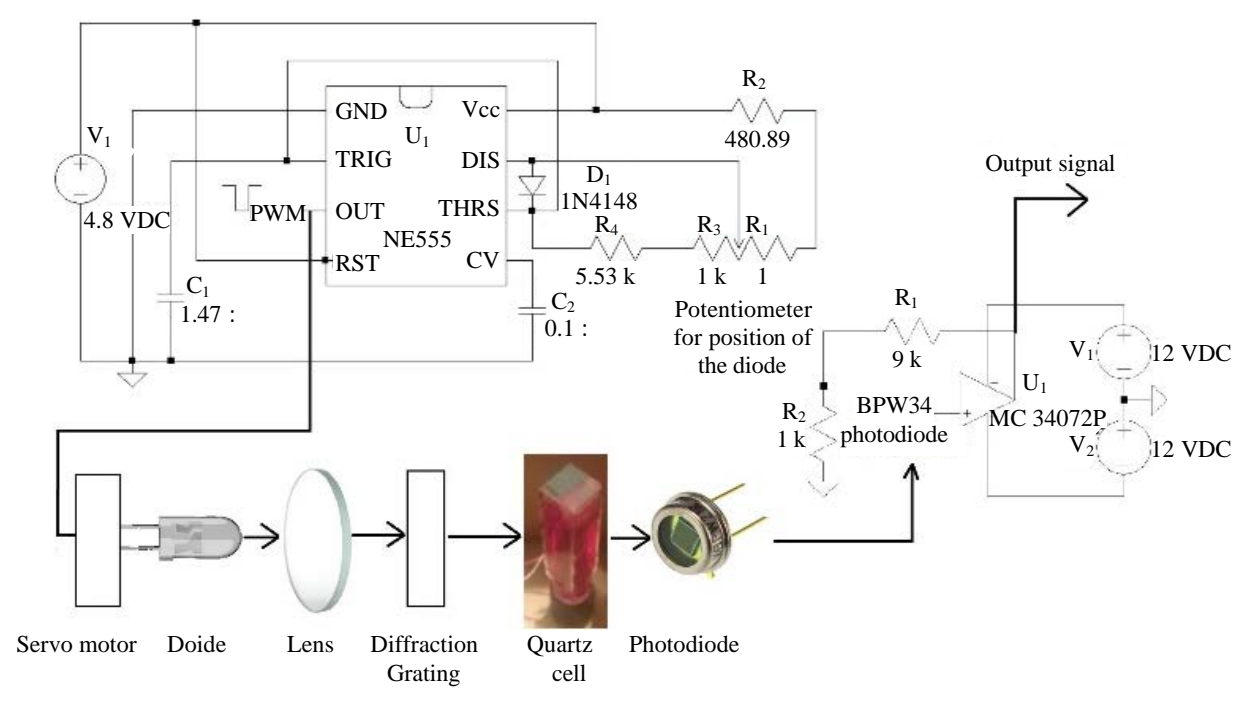

Fig. 1: Complete scheme of the project
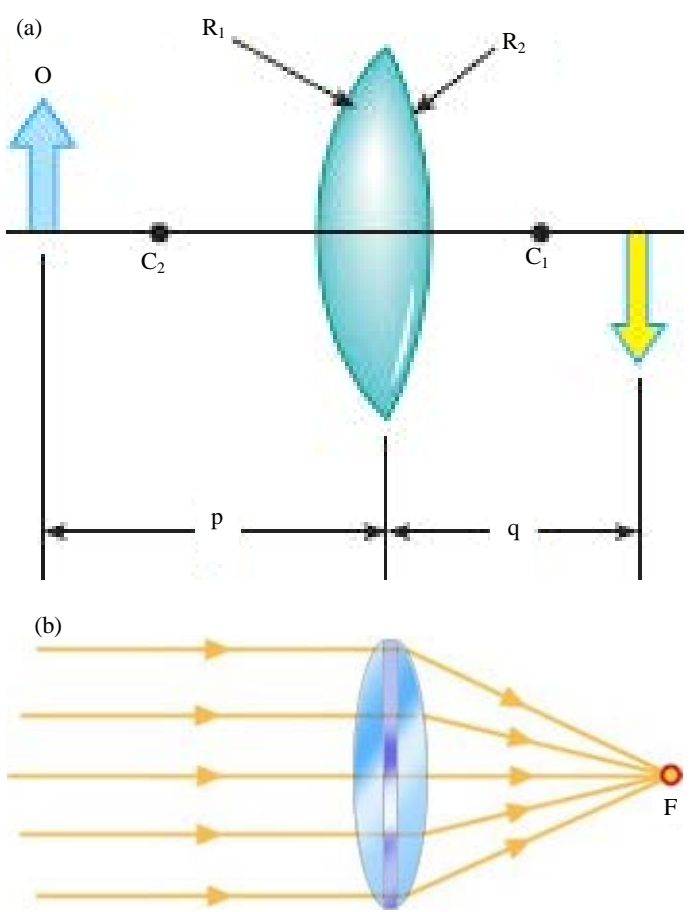

Fig. 2: Behavior of the lens to the incidence of light: a) Distances required by the experiment and b) Input, output and focus point of light through the lens

Figure 2 shows the point $\mathrm{F}$ as the focal distance of the lens and it is calculated as in Eq. 1. In Fig. 2, the points $\mathrm{p}$ and $\mathrm{q}$ correspond to the ranges given in Fig. 2a. This light coming out of the lens is focused on a diffraction grid to be able to separate the white light in the visible spectrum:

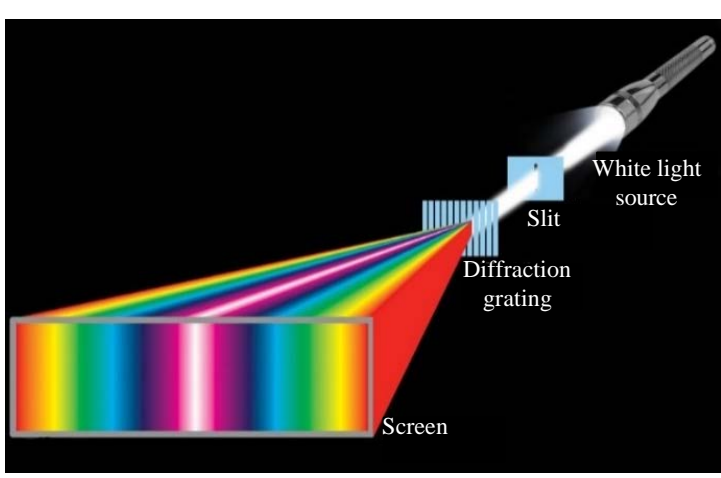

Fig. 3: Diffraction of white light

$$
\frac{1}{\mathrm{f}}=\frac{1}{\mathrm{p}}+\frac{1}{\mathrm{q}}
$$

Diffraction: Is a phenomenon that occurs when the flat light waves pass through a small opaque aperture that acts as a point source of light. In this research, a diffraction grating slide with 1000 lines/mm was used, to obtain a precise definition of the colors. When the light strikes the grid each wavelength is dispersed with a different angle, following Eq. 2. The term d represents the width of the opaque aperture what in this case was considered as $0.000001 \mathrm{~m}$ and is the angle of the diffracted light:

$$
\mathrm{dSen}=\mathrm{n}
$$

Figure 3 shows how white light diffraction occurs. Figure 3 shows how the grid separates the colors at different angles. These angles depend on the wavelength, for example, the red color has an average of $699 \mathrm{~nm}$, this 
angle is $43.63^{\circ}$. Other colors such as violet have an average of $404 \mathrm{~nm}$ and its angle is $23.82^{\circ}$. Figure 3 is theoretical but in practice it is possible to use precision elements and in optimal environmental conditions. To complete the optical component of the spectrophotometer, each color of the visible spectrum is passed through a slit that brings the light directly to each tested sample, to analyze the absorbance and transmittance.

Transmittance: The formula that allows having a measurement of the transmittance can be expressed as in Eq. 3 which is measured as the relation between the input and output intensity of light. The term I is the quantity of light that passes through the sample and the term $I_{0}$ is the quantity of light that reaches the surface of the sample. This expression is used to measure the transmittance of the different samples using the spectrophotometer that generates output voltage signals for each substance used :

$$
\mathrm{T}=\frac{\mathrm{I}}{\mathrm{I}_{0}}
$$

Absorbance: Absorbance is the property of a substance to absorb radiation and it can be expressed as in Eq. 4. Note that the transmittance and absorbance are derivates from the same concept, being the absorbance equal to the negative expression of the logarithm of the transmittance. This expression will be used in the absorbance measurements of the different samples using the spectrophotometer that generates output voltage signals for each substance used:

$$
A=-\log T
$$

Materials and specifications required for the construction of the spectrophotometer: This subsection presents the description of the materials and specifications required for the construction of the spectrophotometer. This spectrophotometer is composed of two general parts, the monochromator which breaks down the white light throughout the visible spectrum and a quartz cell with a sensoramplifier set which is used to analyze the transmitted light. All the materials, the design of the system, the actuator and required integrated circuits are included in the detailed descriptions.

Materials: Table 1 shows the required elements for the construction of the spectrophotometer and the characteristics of those used in the project.
Table 1: Elements used for the spectrophotometer

\begin{tabular}{ll}
\hline Elements & References \\
\hline LED & $1 \mathrm{~W}$ \\
Servomotor & SG90 \\
Timer & 555 \\
Trimmers & The maximum value of $10 \mathrm{~K} \Omega$ \\
Capacitors & $1,0.47,0.1 \mathrm{uF}$ \\
Potentiometer & $1 \mathrm{k} \Omega$ \\
Resistances & 2.2 and $1 \mathrm{k} \Omega$ \\
Diode & $1 \mathrm{n} 4004$ \\
Regulated source & Ref: TPR3005T-3C, $030 \mathrm{~V} \mathrm{DC}, 05 \mathrm{~A}$ \\
Converginglens & Diameter: $5 \mathrm{~cm}$, Focus: $5 \mathrm{~cm}$ \\
Diffractiongrid & 1000 lines $/ \mathrm{mm}$ \\
Cell forthequartz & $1 \times 1 \times 6 \mathrm{~cm}$ \\
Photodiode sensor & BPW 34 \\
Operational amplifier & MC $34072 \mathrm{P}$ \\
Covers of MDF & External cover design $3 \mathrm{~mm}$ \\
\hline
\end{tabular}

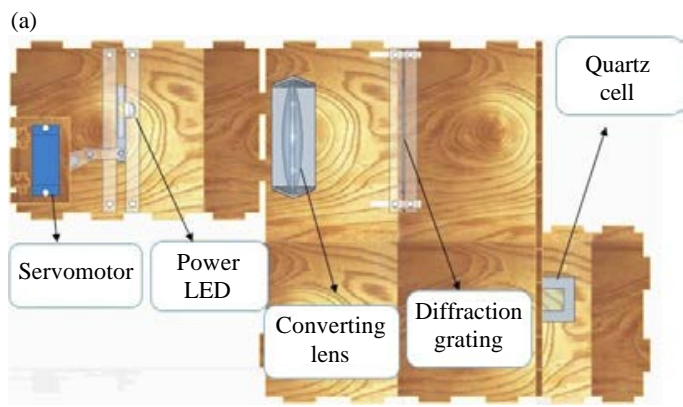

(b)

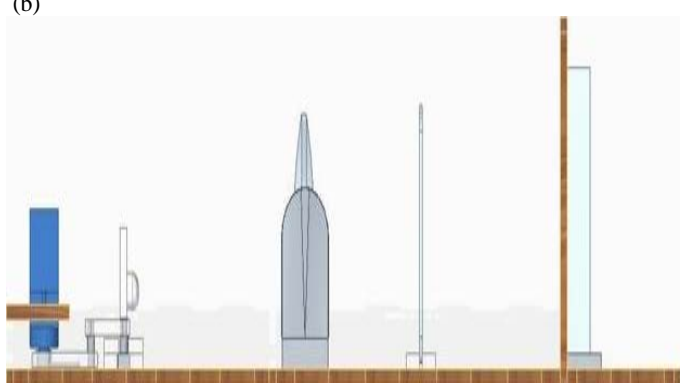

Fig. 4: Spectrophotometer designed for test: a) Upper view and b) Lateral view

Spectrophotometer: The complete built system to measure the colors of the samples is shown in Fig. 4. Figure 4 shows the design of the low-cost spectrophotometer with a servomotor (blue piece) that moves the LED (white section) with some rails and couplings. The light generated by the LED is directed to the converging lens that redirects it to the diffraction grid where the light breaks down throughout the visible spectrum (colors from red to violet) and redirected to a small thickness slit. With the diffraction angles the distance between the diffraction grid and the small slit is calculated.

With the move of the output axis of the servomotor, the LED moves and each color of the spectrum passes 
through the slit to measure the waveform that each color produce in the sample. On the other side of the slit, there is a quartz cell, in which each sample with a specific color is analyzed. Figure 4 shows the space where the quartz cell is located, the BPW 34 luminosity sensor is placed in the face of the cell where the light comes out and this is connected to a voltage amplifier circuit because the voltage delivered only by the sensor is very low and is harder to read.

Servomotor: A servomotor is an electric motor with the capacity to locate in any position within its operating range and to remain in that position. This servomotor has a set of enlarges and a control card which is used to strike the lights into the samples. Servomotors operate by Pulse Width Modulation (PWM) and must be fed with an electric current and also the PWM signal. Usually, these motors work at $50 \mathrm{~Hz}(20 \mathrm{msec})$ and with a duty cycle between 8 and 13\%. Figure 5 shows the duty cycle in a PWM and more precisely the signals in for the operation of a servomotor.

The duration of each pulse is interpreted by the servomotor as positioning commands. When the pulse is $1.5 \mathrm{msec}$, the servomotor maintains its normal position in origin. When the pulse drops to $0.5 \mathrm{msec}$, the servomotor moves $90^{\circ}$ to the left from the origin and when the pulse increase to $2.5 \mathrm{msec}$ the servomotor moves $90^{\circ}$ to the right from the origin. In this research with the help of a potentiometer, the duty cycle can be adjusted to locate the output shaft of the servomotor in various positions. This setting is essential because using rails and couplings, the LED can have a linear movement. This shifting movement is necessary to ensure that the light source is always at the same distance from the lens and thus take more precise measurements.

Integrate circuit: Figure 6 shows the required circuit to control the duty cycle and the frequency. To perform some variations in the duty cycle, an integrated circuit 555 also known as timer 555 was used. When this integrated circuit has a specific voltage value and using some resistances and capacitors, the desired frequency and duty cycle is generated. From equation provided in the datasheet, the resistances and capacitance required for the circuit are: $\mathrm{R}_{1}=480 \Omega, \mathrm{R}_{2}=5.53 \mathrm{k}$ and $\mathrm{C}_{1}$ with $\mathrm{a}$ value of $1.47 \mathrm{uF}$. Between $R_{1}$ and $R_{2}$, a potentiometer is placed to change the duty cycle of the integrated circuit. This assembly is attached to the servomotor by means of the port OUT of the timer, receiving the output that delivers the PWM. The power and ground connections of the servomotor are made separately. Typically, these motors are powered by a voltage value between 4.8 and $5.2 \mathrm{~V}$.

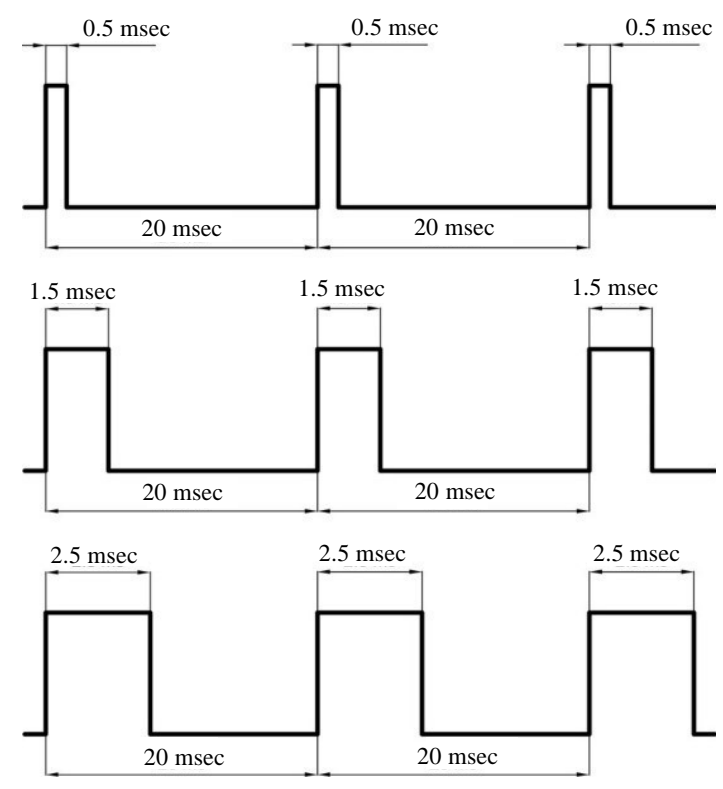

Fig. 5: Duty cycle of the servomotor

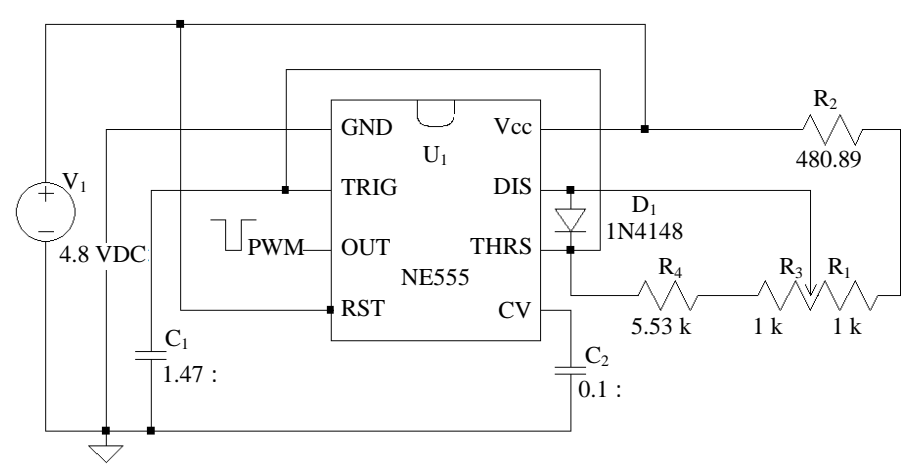

Fig. 6: An integrated circuit to achieve the desired frequency and duty cycle 


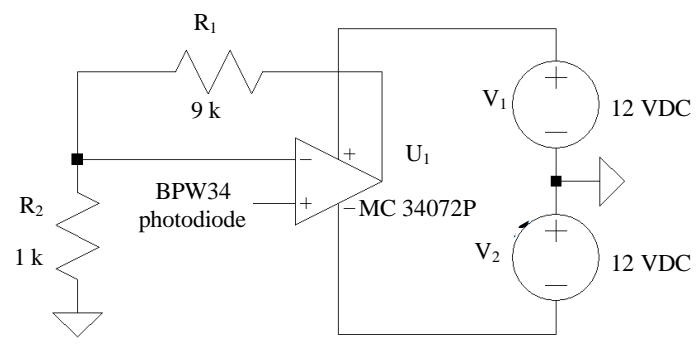

Fig. 7: Amplifier circuit used to improve the spectrophotometry

Voltage amplifier circuit: As the intensity of light that reaches the sensor is very low, the voltage that generates it is also very low. For that reason, to measure that signal with a multimeter or oscilloscope the signal must be amplified. Thus, this requires an MC $34072 \mathrm{P}$ operational amplifier and two resistances; these components form the voltage amplifier circuit. Figure 7 shows the complete amplifier circuit and the amplification value can be calculated by using Eq. 5 :

$$
\mathrm{V}_{0}=\mathrm{V}_{\mathrm{i}} *\left[1+\frac{\mathrm{R}_{2}}{\mathrm{R}_{1}}\right]
$$

where, $V_{\text {in }}$ is the voltage that the sensor measures and $V_{\text {out }}$ is the value of the amplified voltage. For this case, the signal must be amplified 10 time and for that the $\mathrm{R}_{2}$ is used as $9 \mathrm{k}$ and $R_{1}$ as $1 \mathrm{k} \Omega$. The amplified output signal is read with an auto-range multimeter for greater accuracy.

Samples selected for the test: Figure 8 shows the quartz cell with the different samples analyzed with the spectrophotometer. These samples represent the different colors for the test: red orange, yellow, green, blue and violet. Each color in the sample is used to identify the voltage signals and the waveform with the electronic spectrophotometer.

Relation between voltage and light intensity: As the objective of the project is to find the absorbance of each sample with the respective wavelength, a relationship must be located between the voltage obtained and the light intensity as shown in Eq. 3 and 4 where the transmittance and absorbance are calculated from light intensity. As shown in Eq. 6, the energy of the wave is inversely proportional to the wavelength, this means that high lambdas as red or orange have less energy than those with low lambdas such as blue or violet:

$$
\mathrm{E}=\frac{\mathrm{hc}}{\text { lambda }}
$$

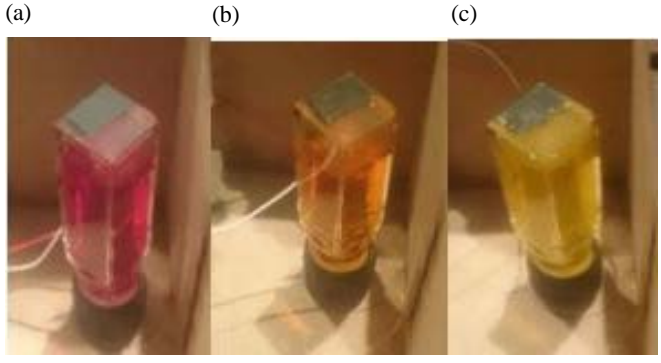

(d)

(e)

(f)

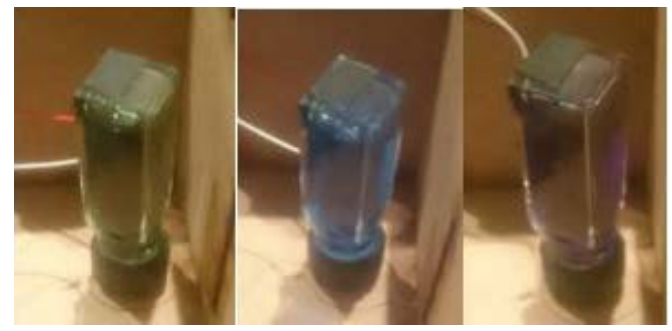

Fig. 8: a-f) Sample of quartz with different pigments used for the test

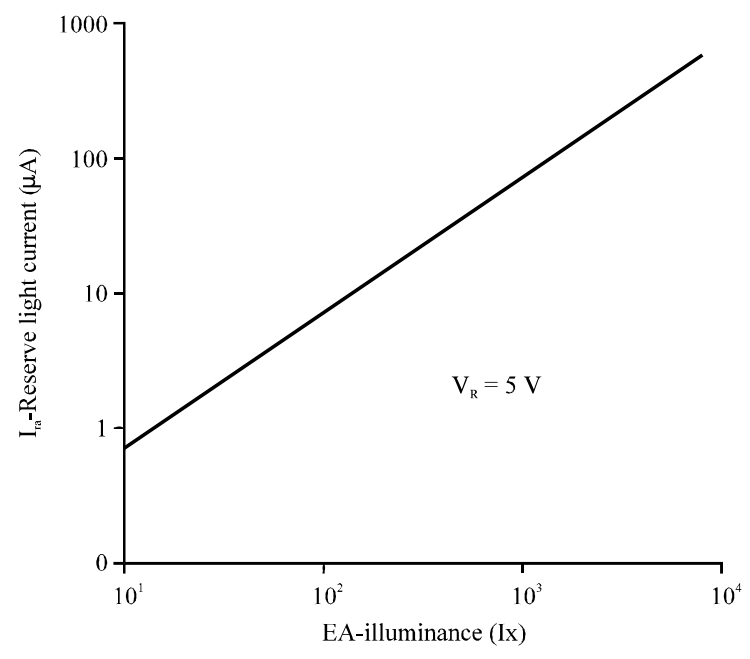

Fig. 9: Relation between the light intensity and the current that passes through the sensors

On the other hand, there is a relation between light intensity and the inverse current that passes through the sensor. As presented in Fig. 9, the light intensity is proportional to current. As the sensor works as a photodiode, the voltage is directly proportional to the current. Thus, the sensor BPW 34 produce a voltage that is directly proportional to the light intensity that strikes on it. This conclusion is essential for subsequent analyses of the transmittance and absorbance as the intensity of light can be determined by measuring the voltage of the sensor (Eq. 3 and 4) with the certainty that the results obtained will be by the reality. 


\section{RESULTS AND DISCUSSION}

This study presents the results obtained by passing each color of the visible spectrum through different solutions of aminobenzene (aniline) and make the light of this result in the sensor. All these experiments are performed to determine the voltage signals generated in the spectrometer and to be able to determine the waveform for each tested substance.

Voltages obtained by spectrophotometry: Figure 10 shows the wavelength with the respective voltage $(\mathrm{mV})$ produced through the spectrum color with no sample. This means that an empty recipient has been placed in the quartz cell to identify the wavelength produced. It is observed that the voltage values are between 447 and $466 \mathrm{mV}$, increasing the values in a linear form in the color spectrum. In the last colors of Fig. 10, the waveform changes especially in the blue color and then returns to its linearity.

Next, the graphs of wavelength against voltage of the experiment carried out are shown but with the cell of quartz filled with a mixture of water with aminobenzene of various colors. It is observed that it is filled with the different colors described as red orange, yellow, green, blue and violet.

When a particular wavelength is passed through the sample, the voltage level rises in areas of color equal to or close that passes through the sensor. Voltage is higher in these areas. It is visible that each sample produces a waveform in the color spectrum and that the voltage magnitude is very particular for that color. The voltage signal is produced with high voltage values in the same color of the spectrum. Although, some samples have high voltage values for similar colors form with the primary colors, for example, the yellow is very present in the colors orange and green, so, the signals remain in high values which does not occur for the red, blue and violet (Fig. 11).

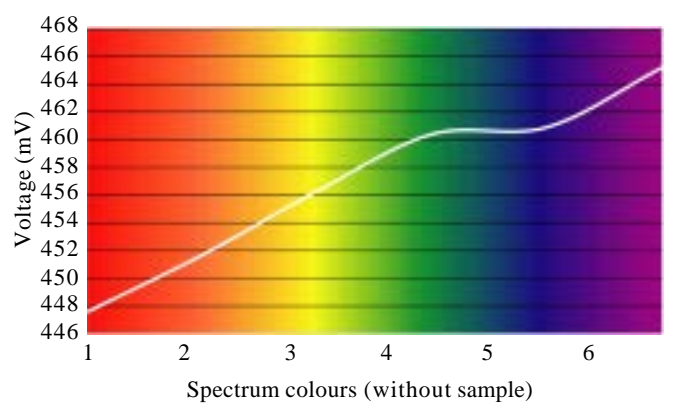

Fig. 10: Wavelength with an empty quartz
Transmittance and absorbance values per sample: Table 2 shows the transmittance and absorbance values of each sample subjected to different wavelengths of the visible spectrum. To do this, a table with the colors of the spectrum to be analyzed and each type of color placed in the quartz cell has been obtained.

When the sunlight or the light of a lamp passes through a translucent object or substance, it is noted that the light passes in its vast majority, depending on the level of clarity of the medium as shown in Table 1. As the samples studied in this research are translucent, most of the light that impacts the samples pass through to measure with the sensor and a tiny portion does not. The results obtained confirm the previous statement, is the percentage of transmittance is $>92 \%$ and the absorbance lower than $2 \%$. These results show the good calibration of the assembly that is presented, thus, guaranteeing optimal results to analyze another type of samples.

Relation between absorbance and wavelength: Figure 12 shows the absorbance and wavelength of the different samples analyzed. This test determines the absorbance in percentage value concerning the different colors of the spectrum. Each line of the graph corresponds to the wavelength emitted with the sample quartz and the color corresponds to the aminobenzene tested.

\begin{tabular}{lrccccc}
\multicolumn{6}{l}{ Table 2: Transmittance and absorbance values for each sample } \\
\hline Samples & \multicolumn{1}{c}{ Red } & Orange & Yellow & Green & Blue & Violet \\
Red & 98.190 & 97.343 & 96.165 & 94.896 & 94.554 & 93.723 \\
Orange & 96.626 & 96.812 & 96.625 & 95.460 & 93.730 & 92.842 \\
Yellow & 97.900 & 98.140 & 98.313 & 97.459 & 95.357 & 93.831 \\
Green & 95.956 & 95.307 & 94.609 & 94.526 & 94.142 & 93.014 \\
Blue & 96.403 & 95.749 & 95.025 & 95.765 & 97.527 & 95.550 \\
Violet & 96.649 & 95.749 & 94.894 & 94.201 & 94.966 & 94.733 \\
Red & 1.993 & 1.992 & 1.988 & 1.983 & 1.977 & 1.976 \\
Orange & 1.985 & 1.986 & 1.985 & 1.980 & 1.972 & 1.968 \\
Yellow & 1.991 & 1.992 & 1.993 & 1.989 & 1.979 & 1.972 \\
Green & 1.982 & 1.979 & 1.976 & 1.976 & 1.974 & 1.969 \\
Blue & 1.984 & 1.981 & 1.978 & 1.981 & 1.989 & 1.980 \\
Violet & 1.985 & 1.981 & 1.977 & 1.974 & 1.978 & 1.977 \\
\hline & & & & & &
\end{tabular}

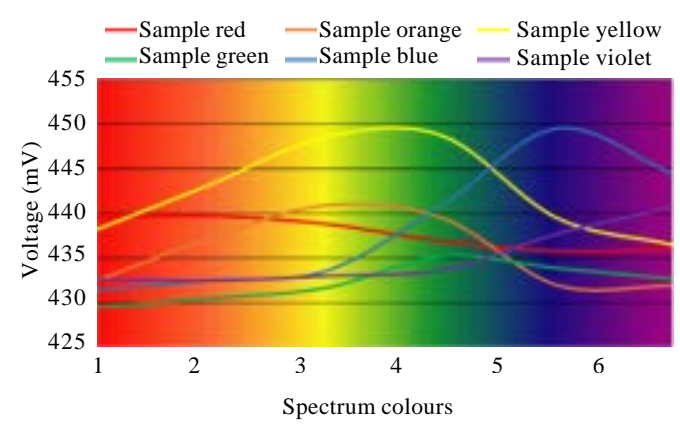

Fig. 11: Wavelength with the different quartzes filled with the aminobenzene of the six main colours 


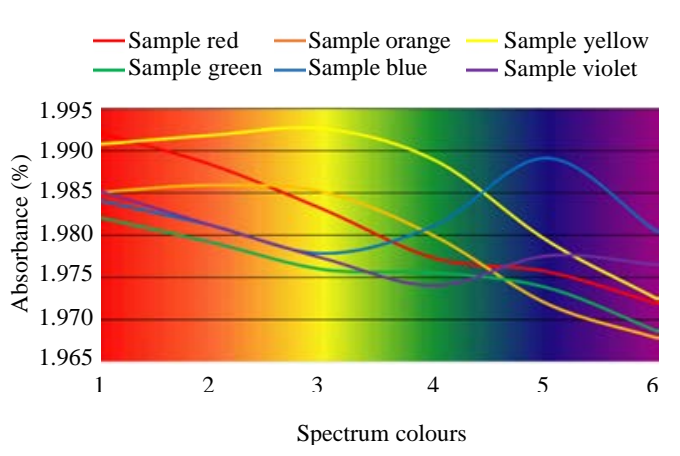

Fig. 12: Absorbance in different samples

The absorbance and transmittance have similar behaviors when the red sample interacts with the visible spectrum. This shows a linear behavior in wavelengths from the red to the green. There is an irregularity in the spectrum between the colors green and blue as in this area the absorbance and transmittance present an increase due to the combination of the red sample with this wavelength. In the orange sample the transmittance and absorbance are greater in wavelengths close to orange and yellow with the rest of lambdas these behave almost linearly.

Similar behavior is noted in the yellow sample, showing an increase in the values of $\mathrm{T}$ and a for wavelengths near the yellow spectrum. In the green sample it is seen that it has a linear behavior in wavelengths corresponding to spectra from red to yellow, there is a little normalization in spectra from yellow to blue and from blue onwards again takes a linear behavior. It is also noted that the blue sample has a higher absorbance at wavelengths near the blue spectrum. The violet sample has a greater absorbance in the red spectrum and a lesser in spectra near the green.

\section{CONCLUSION}

This study presented the application of spectrophotometry to measure transmittance and absorbance of light applied to water with aminobenzene of different colors. The test based on creating a low-cost spectrophotometer that produces voltage signals that can be analyzed to determine the amount of light that passes each sample and compare with the wavelengths of the visible spectrum impact. A unique structure in $\mathrm{MDF}$ was built with the necessary focal distances of the lens and the diffraction angles, optimizing the light beam that strikes the sample. This research showed that the low-cost spectrophotometer designed for the test complies with the conditions to ensure an optimal analysis of the samples. The results show that when wavelength decreases, the energy increases which is represented by the voltage measured by the sensor. When a wavelength impact on the sample with the same colour, the light coming out of this will intensify concerning the other colours of the visible spectrum. This could be demonstrated by the colour diagram in the study in which a relative voltage increase is noted in areas where the similar sample and lambda colours are combined.

With the characteristics given by the manufacturer of the light sensor, it was possible to obtain a direct relationship between the measured voltage and the light intensity. All this allowed replacing the transmittance and absorbance formulas with new voltage values measured in the tests. The transmittance measured in the samples is always above $92 \%$ and the absorbance below $2 \%$. Besides, there is a high relation between absorbance and different wavelengths, the different samples of aminobenzene adsorb and transfer the different wavelengths of the visible spectrum differently. In future research the tests will be performed to identify different substances improving the identification with the voltage magnitudes and the waveforms. Besides, the control of the LED can be improved to locate a specific lambda in the slit with digital commands and obtaining real-time graphics of the relationships between absorbance and wavelength.

\section{ACKNOWLEDGEMENTS}

This research was supported by the Universidad Nacional de Colombia, Sede Medellin, under the projects HERMES-34671 and HERMES-36911. The researchers thank to the School of Physics and the Department of Electrical Energy and Automation of the Universidad Nacional de Colombia for the valuable help to conduct this research.

\section{REFERENCES}

Burnum-Johnson, K.E., E.S. Baker and T.O. Metz, 2017. Characterizing the lipid and metabolite changes associated with placental function and pregnancy complications using ion mobility spectrometry-mass spectrometry and mass spectrometry imaging. Placenta, 60: S67-S72.

Garrett, T.A., 2017. Major roles for minor bacterial lipids identified by mass spectrometry. Biochim. Biophys. Acta BBA. Mol. Cell Biol. Lipids, 1862: 1319-1324. 
Gloess, A.N., C. Yeretzian, R. Knochenmuss and M. Groessl, 2018. On-line analysis of coffee roasting with ion mobility spectrometry-mass spectrometry (IMS-MS). Intl. J. Mass Spectrom., 424: 49-57.

Ketha, S.S., R.J. Singh and H. Ketha, 2017. Role of mass spectrometry in clinical endocrinology. Endocrinol. Metab. Clinics N. Am., 46: 593-613.

Petruci, J.F.D.S., M.G. Liebetanz, A.A. Cardoso and P.C. Hauser, 2017. Absorbance detector for high performance liquid chromatography based on a deep-UV light-emitting diode at $235 \mathrm{~nm}$. J. Chromatogr. A., 1512: 143-146.
Rojas, F.S., C.B. Ojeda and J.M.C. Pavon, 2013. Spectrophotometry | Biochemical Applications. In: Reference Module in Chemistry, Molecular Sciences and Chemical Engineering Encyclopedia of Analytical Science, Reedijk, J. (Ed.). Elsevier, Amsterdam, Netherlands, pp: 205-213.

Takamoto, A., K. Sugahara, T. Shibahara, A. Katakura and K. Matsuzaka et al., 2013. Screening for oral mucosal diseases by a portable spectrophotometer: Comparison between color difference and epithelial thickness. J. Oral Maxillofacial Surg. Med. Pathol., 25: 314-327. 